Annuaire suisse de politique de développement

9 | 1990

Annuaire Suisse - Tiers Monde 1990

\title{
V. Coopération au développement
}

\section{(2) OpenEdition}

1 Journals

Édition électronique

URL : http://journals.openedition.org/aspd/1306

DOI : $10.4000 /$ aspd. 1306

ISSN : 1663-9669

Éditeur

Institut de hautes études internationales et du développement

\section{Édition imprimée}

Date de publication : 1 janvier 1990

Pagination : 112-134

ISSN : 1660-5934

\section{Référence électronique}

"V. Coopération au développement », Annuaire suisse de politique de développement [En ligne], 9 | 1990 mis en ligne le 28 mars 2013, consulté le 08 septembre 2020. URL : http://journals.openedition.org/ aspd/1306 ; DOI : https://doi.org/10.4000/aspd.1306 


\section{Coopération au développement}

\section{Aide publique au développement en 1988}

\subsection{Généralités}

L'aide publique au développement de la Suisse suit une courbe régulièrement ascendante comme le montre le tableau 17. Ces dernières années, les dépenses ont passé de 344 millions de francs $(0,21 \%$ du PNB) en 1979 à 857 millions $(0,31 \%$ du PNB) en 1988 . Mais la Suisse se situe encore en-dessous de la moyenne de 18 pays du CAD (1) qui était de 0,35\% du PNB en 1988 (2). Sur proposition du Département fédéral des finances, les dépenses pour la coopération au développement devraient dorénavant plafonner à un maximum de $0,32 \%$ du PNB.

Les oeuvres d'entraide suisses estiment pour leur part que ce plafonnement peut signifier un recul dramatique des activités de la coopération au développement. Au vu de l'endettement des pays en développement, de l'effondrement des prix des matières premières, des problèmes d'environnement et des mouvements de migratiorı et de fuite, un besoin bien plus grand de crédits pour l'aide au développement se fait sentir. Les oeuvres d'entraide demandent au Conseil fédéral d'adapter à moyen terme les prestations de la Suisse à la moyenne de $0,5 \%$ du PNB consacré au développement par la Communauté européenne. Dans sa séance du 16 août 1989, l'ensemble du Conseil fédéral a rejeté la proposition du Département fédéral des finances et a décidé de poursuivre l'augmentation de l'aide au développement, ce qui aura des répercussions sur le plan financier 1991-1993.

La Confédération finance la plus grande partie de l'aide publique au développement, à raison de 840,8 millions de francs soit le $98,1 \%$; les cantons et les communes représentent en 1988 une part de 1,9\% avec 16,7 millions de francs. La part des dépenses pour l'aide au développement représente le $3,2 \%$ des dépenses totales de la Confédération. 
Tableau $\mathrm{N}^{\circ} 17$

\begin{tabular}{|c|c|c|c|}
\hline \multicolumn{4}{|c|}{ Evolution de l'aide publique au développement 1979-1988 } \\
\hline 1979 & 344 Mio Fr. & 1984 & 617 Mio Fr. \\
\hline 1980 & 412 Mio Fr. & 1985 & 677 Mio Fr. \\
\hline 1981 & $453 \mathrm{Mio} F r$. & 1986 & 725 Mio Fr. \\
\hline 1982 & 490 Mio Fr. & 1987 & 767 Mio Fr. \\
\hline 1983 & 574 Mio Fr. & 1988 & 857 Mio Fr. \\
\hline
\end{tabular}

La plus grande partie (76\%) des dépenses totales se fait sous forme d'aide bilatérale. Cette forme d'aide est en règle générale moins controversée en Suisse que les dépenses faites dans le cadre multilatéral (24\%). La raison en est que l'utilisation des crédits pour des projets et des programmes bilatéraux peut être mieux contrôlée que le cofinancement de grands programmes multilatéraux. Depuis des années, la communauté de travail de Swissaid, Action de Carême, Pain pour le Prochain et Helvetas critique le fait que le cofinancement de la filiale de la Banque mondiale, l'AID, soit prise dans le compte de l'aide bilatérale au niveau statistique. En 1988, cela représentait 52,7 millions de francs bien que la manière de pratiquer de l'AID soit nettement multilatérale. La raison principale de la critique de la communauté de travail est avant tout le manque d'un concept sur la forme de l'aide multilatérale.

Au sein de la DDA, la séparation entre aide multilatérale et bilatérale est illustrée par l'exemple de la production de la soie en Inde (rapport annuel, p.18):

“Petit pays, la Suisse n'est guère en mesure de financer seule un projet couvrant un vaste périmètre géographique. Une remarque à considérer dans le cas de notre aide à la sériciculture indienne. Dans nos petits projets bilatéraux, nous pouvons cependant tester de nouvelles techniques, de nouvelles formes de commercialisation; nous pouvons par exemple procéder à des sondages pour voir dans quelle mesure la sériciculture est également possible dans des régions arides et peu exploitées.

Les grands projets multilatéraux ne laissent guère de place aux expérimentations, car ils font appel à des montants financiers par trop importants. Quand nous participons à la réalisation de projets semblables, nous sommes cependant en mesure de les faire profiter des expériences tirées de notre coopération bilatérale. Les programmes intéressant de vastes territoires peuvent en être ainsi qualitativement améliorés." 
La coopération suisse au développement inclut les cinq domaines suivants: la coopération technique, l'aide financière, les mesures de politique économique et commerciale, l'aide alimentaire et l'aide humanitaire. La plus grande part est consacrée à la coopération technique avec 45\%, en 1988 (voir tableau 18).

Tableau $\mathrm{N}^{\circ} 18$

Dépenses pour l'aide publique au développement 1987/1988

\begin{tabular}{|c|c|c|c|c|c|c|}
\hline & \multicolumn{4}{|c|}{1988} & \multicolumn{2}{|c|}{1987} \\
\hline & $\begin{array}{c}\text { bilatérale } \\
\text { mio fr. }\end{array}$ & $\begin{array}{c}\text { multi- } \\
\text { latérale } \\
\text { mio fr. }\end{array}$ & $\begin{array}{l}\text { total } \\
\text { mio fr. }\end{array}$ & $\%$ & $\begin{array}{l}\text { total } \\
\text { mio fr. }\end{array}$ & $\%$ \\
\hline Coopération technique & 292 & 92 & 384 & 45 & 330 & 43 \\
\hline $\begin{array}{l}\text { Aide financière } \\
\text { Mesures économiques }\end{array}$ & 97 & 67 & 164 & 19 & 195 & 25 \\
\hline et commerciales & 110 & - & 110 & 13 & 68 & 9 \\
\hline Aide alimentaire & 33 & 25 & 58 & 7 & 56 & 7 \\
\hline Aide humanitaire & 105 & 12 & 117 & 14 & 97 & 13 \\
\hline Non classé & 20 & 10 & 30 & 3 & 26 & 4 \\
\hline Remboursement de prêts & -6 & - & -6 & -1 & -5 & -1 \\
\hline Total & 651 & 206 & 857 & 100 & 767 & 100 \\
\hline En pourcentage du PNB & & & 0,31 & & 0,29 & \\
\hline
\end{tabular}

Un nouvel instrument

Au cours de la période couverte par l'Annuaire, un nouvel instrument de coopération a été appliqué: les paiements compensatoires pour les pertes de recettes d'exportation dans le commerce de matières premières avec la Suisse. On a accordé à six pays africains (le Soudan, la Tanzanie, le Togo, le Tchad, l'Ouganda et la République centrafricaine) une aide à fonds perdu de 15,8 millions de francs compensant des baisses de prix sur des produits exportés en Suisse. Ces pays, parmi les pays en développement les plus pauvres, ont subi en 1986 et 1987 les baisses de recettes d'exportation les plus importantes dans leur commerce avec la Suisse. Ces paiements compensatoires sont avant tout versés pour les matières premières qui ont baissé. (La Communauté européenne a conclu il y a quelques années déjà un accord "Stabex" avec des pays 
en développement, qui permet de compenser ce genre de pertes). (Voir à ce sujet sous "CNUCED/Financement compensatoire" au chapitre "Négocations internationales").

\section{Coopération au développement et environnement}

Vu la destruction croissante de l'environnement au niveau planétaire, les projets et les programmes dans le cadre de la coopération au développement sont examinés de plus en plus sous l'aspect de leur impact sur l'environnement. Au centre des débats se trouve la mise en pratique de politiques et de concepts dans des projets pratiques de coopération au développement. La priorité est mise sur les projets permettant aux pays en développement de mettre en place leurs propres mesures de protection de l'environnement.

En 1988, deux rencontres placées sous le thème de l'environnement ont eu lieu dans le cadre du comité de développement de l'OCDE (DAC) auxquelles la Suisse a participé activement. Une liste de contrôle a été mise au point qui permet aux responsables d'estimer l'impact sur l'environnement de projets de développement. En outre, les mesures prises par certains pays dans ce domaine ont été discutées.

Un des points centraux de ce travail est de renforcer la conscience sur les tenants et aboutissants de la destruction de la nature, de mettre en place une politique de protection de l'environnement au sens large et d'intégrer ses coûts dans la rentabilité d'un projet (rapport 1988 sur la participation de la Suisse aux activités internationales dans le domaine de l'environnement, p.9). II s'agit de tenir compte du potentiel de ressources dans la planification, les décisions, l'exécution et l'évaluation des projets. Dans le rapport, il est clairement déclaré qu'il ne suffisait pas d'amener des corrections, d'améliorer un projet au vu de dommages constatés par une étude d'impact sur l'environnement, mais qu'il fallait développer et encourager les activités qui régénèrent l'environnement.

Dans le cadre du comité interdépartemental pour la coopération au développement international et l'aide humanitaire, la DDA et l'OFAEE ont mis au point un guide méthodologique de l'impact sur l'environnement des activités de la coopération suisse au développement avec l'assistance de l'Office fédéral de l'environnement, des forêts et du paysage. En 1989/90, une phase de test-pilote de 18 mois est en cours pour la mise en place d'instruments et de règlements administratifs pour l'étude d'impact.

Une question ordinaire (Seiler, conseiller aux Etats) du 15 juin 1989 concernant les études d'impact sur l'environnement de projets dans les pays en voie de développement demandait au Conseil fédéral une réponse aux questions sulvantes: "Est-ce qu'une étude d'impact sur l'environnement est faite systématiquement pour tous les projets de développement que la Suisse soutient dans le Tiers-Monde? Si oui, depuis quand? Est-ce que les programmes sectoriels et les programmes de restructuration ainsi que les projets inclus dans le cadre du crédit pour les mesures économiques et commerciales comme les crédits mix- 
tes et la garantie contre les risques à l'exportation dans les pays en développement les plus pauvres y sont également soumis?"

Le Conseil fédéral a répondu le 13 septembre 1989 en soulignant que les programmes nationaux et sectoriels n'étaient pas systématiquement soumis à une étude d'impact sur l'environnement, mais que cela dépendait de la situation des ressources naturelles. Dorénavant pourtant, une analyse des risques sur l'environnement devrait venir compléter tout programme national. Depuis plusieurs années déjà, on tient compte des effets sur l'environnement des projets de développement financés par le biais des crédits mixtes; depuis 1988, ils sont analysés systématiquement pour tout projet.

Dans les pays les moins avancés, les petits projets couverts par la GRE sont analysés quant à leurs effets sur l'environnement, si la nécessité se fait sentir, et ce, en collaboration avec le service du développement de l'OFAEE.

En ce qui concerne les programmes d'ajustement structurel (sous la direction du FMl et de la Banque mondiale), le Conseil fédéral fait remarquer que l'appréciation des effets sur l'environnement des programmes d'ajustement structurel n'est pas simple et très controversée. Selon lui, "les faiblesses structurelles, le développement démographique, l'endettement et la pauvreté provoquent dans de nombreux pays en développement une charge plus lourde sur l'environnement. C'est pourquoi l'amélioration des conditions économiques générales peut aussi contribuer à long terme à maintenir l'environnement". Plus loin: "C'est justement dans une perspective de maintien de l'environnement que nous devons être conscients que l'ajustement structurel implique une responsabilité globale et qu'il faut agir plus directement qu'auparavant contre les inégalités mondiales et cela, chez nous également."

L'intention de la coopération au développement est de soutenir les efforts propres des pays dans leur mise en place de mesures en faveur de l'environnement, afin que l'étude d'impact sur l'environnement ne devienne pas une condition pour l'octroi de crédits pour le développement, mais plutôt que cela devienne un procédé automatique dans les pays eux-mêmes lors de la planification de projets de développement.

\section{Thèmes des conférences annuelles: les ONG et l'endettement}

Le thème de la conférence annuelle de la DDA de 1988 était consacré aux organisations non-gouvernementales (ONG) dans le Tiers-Monde et leur rôle dans la coopération au développement. Une collaboration plus étroite avec les ONG dans les pays partenaires est nécessaire pour atteindre l'objectif de la coopération suisse au développement qui est de soutenir les groupes de la population les plus défavorisés dans leurs efforts de développement. Cela renforce la position des organisations privées dans les pays en développement face aux gouvernements.

Le thème de la conférence annuelle de 1989 était la dette du Tiers-Monde. L'ancien ministre du plan bolivien, Fernando Romero, était le conférencier invité 
Tableau $\mathrm{N}^{\circ} 19$

\section{Flux financiers nets de la Suisse vers les pays en développement de 1985 à 1987}

\begin{tabular}{|c|c|c|c|}
\hline & \multicolumn{3}{|c|}{ en millions de francs } \\
\hline $\begin{array}{l}\text { Aide publique au développement (APD) } \\
\text { Dons des oeuvres privées d'entraide } \\
\text { Autres apports du secteur public } \\
\text { Flux de capitaux privés } \\
\text { dont: Investissements directs } \\
\text { Crédits à l'exportation } \\
\text { Emprunts sur le marché suisse } \\
\text { des capitaux } \\
\text { Flux bancaires }\end{array}$ & $\begin{array}{r}681,2 \\
132,5 \\
-12,5 \\
5290,9 \\
1200,6 \\
-340,8 \\
4128,2 \\
302,9\end{array}$ & $\begin{array}{r}718,3 \\
119,4 \\
-12,6 \\
1628,7 \\
853,9 \\
-597, .2 \\
\\
917,6 \\
454,4\end{array}$ & $\begin{array}{r}767,3 \\
140,1 \\
-14,6 \\
-3353,4 \\
-495,7 \\
-1270,5 \\
-1320,1 \\
-267,1\end{array}$ \\
\hline \multirow[t]{2}{*}{$\begin{array}{l}\text { Total des flux financiers nets } \\
\text { publics et privés }\end{array}$} & 6092,1 & 2453,8 & $-2460,6$ \\
\hline & \multicolumn{3}{|c|}{ en pourcentage du PNB } \\
\hline $\begin{array}{l}\text { Aide publique au développement (APD) } \\
\text { Dons des oeuvres privées d'entraide } \\
\text { Autres apports du secteur public } \\
\text { Flux de capitaux privés }\end{array}$ & $\begin{array}{r}0,28 \\
0,05 \\
-0,01 \\
2,19\end{array}$ & $\begin{array}{r}0,28 \\
0,05 \\
0,63\end{array}$ & $\begin{array}{r}0,29 \\
0,05 \\
-0,01 \\
-1,16\end{array}$ \\
\hline $\begin{array}{l}\text { Total des flux financiers nets } \\
\text { publics et privés }\end{array}$ & 2,51 & 0,96 & $-0,83$ \\
\hline
\end{tabular}

"Alde publique au développement": elle représente l'ensemble des flux financiers vers les pays en développement et les institutions multilatérales provenant de fonds publics. Ces prestations sont fournies dans le but essentiel de favoriser le développement économique de ces pays et sont accordées à des conditions de faveur.

"Les dons des oeuvres privées d'entraide" comprennent les prestations au titre de la coopération au développement et des actions d'urgence des organisations privées sans but lucratif.

"Les autres apports du secteur public" se réfèrent à tous les autres moyens mis à disposition des pays en développement par les collectivités publiques mais qui ne sont pas assortis de conditions de faveur.

"Flux de capltaux privés": ce sont tous les moyens mis à disposition par l'économie privée aux conditions du marché pour les pays en développement, les investissements directs, les crédits à l'exportation, les souscriptions d'emprunts et les prêts, qui sont accordés par des banques établies en Suisse à des pays en développement (avoirs à long terme). Lorsqu'ils ne représentents pas des remboursements, les "contre-flux" qui résultent des opérations menées par des personnes résidant dans les pays en développement ne sont pas pris en compte.

PNB 1985: 241355 millions de fr.

PNB 1986: 254510 millions de $\mathrm{fr}$.

PNB 1987: 266270 millions de fr.

Source: Rapport annuel de la DDA 1988 
pour parler en tant que représentant d'un pays confronté à ce problème. La Bolivie est citée comme modèle dans le continent latino-américain en ce qui concerne l'effort de solution du problème de la dette. En 1988, la Suisse a accordé à la Bolivie un crédit de 5 millions de francs pour le rachat de titres de créances avec des rabais de $90 \%$.

\section{Flux nets de capital négatifs}

Si l'on compare les dépenses privées et publiques pour l'aide au développement au flux de capitaux privés, un tournant dramatique se dessine de 1985 à 1987 en ce qui concerne les flux nets de capitaux de la Suisse vers les pays en voie de développement. Si, en 1985, quelque 6 milliards de francs s'en allaient dans les pays en développement, en 1987, ces mêmes pays ont payé 2 milliards de francs nets à la Suisse sous forme de remboursements de crédits et de paiements d'intérêts. Face à cet état de fait, l'aide au développement privée et publique suisse est très modeste (voir tableau 19 ainsi que les tableaux 2.1.A et 2.3.A de la partie statistique pour l'année 1988).

\subsection{Alde bilatérale}

En 1988, un montant total de 651 millions de francs a été consacré à l'aide bilatérale. Ce chiffre englobe toutes les dépenses bilatérales faites à la fois dans le cadre de la coopération technique (part la plus importante), l'aide financière, les mesures économiques et commerciales ainsi que l'aide humanitaire, y compris l'aide alimentaire. En Suisse, on accorde la préférence à l'aide bilatérale. En plus d'un meilleur contrôle en ce qui concerne l'utilisation des moyens, c'est le contact direct avec les pays partenaires qui joue un rôle important. L'aide est à fonds perdu et non-liée.

\section{Répartition selon le niveau de revenu}

Le groupe des pays les moins avancés ( les PMA, une liste de 42 pays en développement les plus pauvres selon l'ONU) a bénéficié d'une aide de 205 millions de francs. Les autres pays à bas revenu (PNB par capita inférieur à 800 dollars) ont reçu 213 millions de francs. 72 millions sont allés à destination des pays à revenu moyen (PNB par capita supérieur à 800 dollars).

\section{Répartition par régions}

En 1988, l'aide bilatérale, sans l'aide humanitaire, se répartissait de la façon suivante dans les pays en développement: Afrique $45,5 \%$ (169,6 millions de francs), Asie 28,4\% (105,8 millions), Amérique latine 16,7\% (62 millions). 
Les principaux pays bénéficiaires d'Afrique ont été en 1988 Madagascar (25,2 millions de francs), le Rwanda (16,5 mio.), la Tanzanie (14,5 mio.), le Mali (12,0 mio.) et le Niger (10,1 millions).

Parmi les pays en développement asiatiques, l'Inde a obtenu 27,9 millions de francs, suivi de l'Indonésie (19,8 mio.), le Népal (18,3 mio.), le Pakistan (8,8 mio.) et le Bhoutan ( 8,4 millions).

En Amérique latine, la Bolivie est un des pays-clés de la coopération suisse au développement. En 1988, 23,1 millions de francs leur ont été attribués. Le Pérou a obtenu 7,7 mio., le Honduras 6,2 mio., le Nicaragua 5,7 mio. et l'Equateur 3,5 millions.

(La partie statistique du présent Annuaire contient la liste des principaux pays bénéficiaires de la coopération suisse bilatérale, de même que d'autres données relatives au volume et au genre d'aides.)

\section{Répartition par secteurs}

Depuis le début de la coopération suisse au développement, l'agriculture et la sylviculture ont constitué le secteur le plus important de soutien avec $30 \%$ des fonds. Le graphique de la page suivante montre la répartition de l'aide dans les différents secteurs pour le continent africain, asiatique et latino-américain. (Une base de $100 \%$ est prise pour chaque continent.)

\subsection{Coopération technique et aide financière}

La coopération technique et l'aide financière ont pour but d'accroître les connaissances et le savoir-faire dans le domaine technique ainsi que d'améliorer la capacité d'utiliser plus rationnellement les facteurs de production. Les moyens utilisés à cet effet sont des projets et des programmes techniques. L'aide financière est soit accordée pour des investissements dans différents secteurs, soit permet de soutenir des programmes de restructuration sectorielle. L'aide financière va souvent de pair avec des projets et programmes de la coopération technique. Cette forme d'aide se fait aussi bien par le biais de mesures bilatérales que multilatérales.

Comme le tableau 18 de "l'aide publique suisse au développement" le montre, 292 millions de francs ont été employés en 1988 par la coopération technique par la voie bilatérale, 92 millions par la collaboration multilatérale. Les dépenses pour l'aide financière se sont élevées à 97 millions de francs au niveau bilatéral et à 67 millions au niveau multilatéral. Les dépenses globales pour la coopération technique et l'aide financière se montent à 548 millions de francs, soit $64 \%$ du budget de l'aide publique au développement. Une partie de cette somme a servi à financer des projets exécutés par la Confédération elle-même. Une autre partie a été versée à titre de contributions à des organisations inter- 
120

ANNUAIRE SUISSE-TIERS MONDE 1990

Répartition sectorielle de l'aide de la cooperation suisse au développement par continents en 1988 (en pourcent)
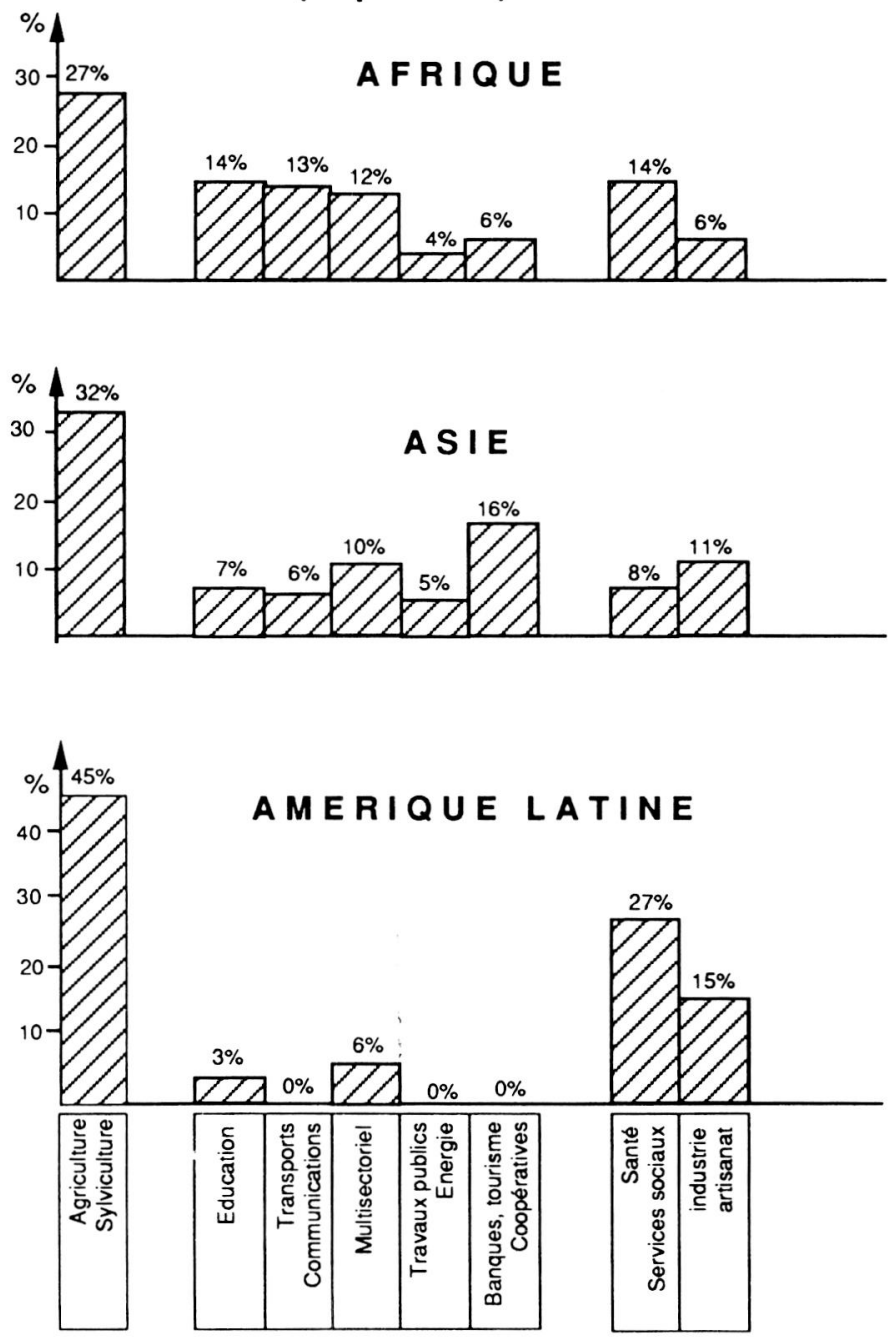

Source : Rapport annuel de la DDA, 1989 
nationales. Enfin un montant de 109 millions a été attribué à des oeuvres privées d'entraide pour qu'elles réalisent leurs propres actions de coopération technique ou des mandats de la Confédération.

Le financement de la formation de boursiers en provenance de pays en développement est par exemple une mesure bilatérale de la coopération technique. En 1988, 405 boursiers ont reçu une aide à la formation ou au perfectionnement, dont 248 originaires d'Afrique, 89 d'Asie, 49 d'Amérique latine et 19 d'Europe.

\subsection{Mesures de politique économique et commerciale}

L'évolution du commerce extérieur suisse avec le Tiers-Monde de 1980 à 1987 indique une diminution de la part du marché des pays en voie de développement dans l'ensemble du commerce extérieur suisse. En 1987, les exportations en direction des pays en développement d'une valeur de 10'634 millions de francs représentaient le $15,8 \%$ de toutes les exportations suisses. En 1980, elles représentaient encore le $18,9 \%$ des exportations suisses avec une valeur de 9'390 millions de francs. La part la plus élevée a été enregistrée en 1982 avec $22 \%$. Au niveau des importations, le $7,5 \%$ de toutes les importations suisses provenait des pays en développement, représentant une valeur de 5'644 millions de francs. En 1980, un montant quasiment identique, 5'495 millions, représentait encore le $9 \%$ de toutes les importations. (Le niveau maximum a été atteint en 1984 avec $9,4 \%$ ).

En 1988 les exportations en direction des pays en développement s'élevaient à 11.942 millions de francs et les importations à 6.300 millions.

Au commerce extérieur de la Suisse avec les pays en développement en diminution font face les efforts de plus en plus nombreux de la coopération suisse au développement sous forme de mesures de politique économique et commerciale. Ces mesures comprennent les aides à la balance des paiements, les crédits mixtes, la promotion commerciale, l'encouragement à l'investissement privé et des mesures dans le secteur des matières premières. En 1988, on a dépensé 108,8 millions de francs, ce qui signifie une nette progression par rapport à 1987 où 67,8 millions avaient soutenu ces mesures. Pour plus de détails, voir le tableau 20.

Les mesures de politique économique et commerciale figurent dans les statistiques sous la rubrique de l'aide bilatérale bien que, dans ce cas également, la Suisse a soutenu des programmes internationaux de caractère multilatéral, en particulier dans le secteur des matières premières.

\section{Aides à la balance des paiements}

Il faut tout d'abord remarquer que les dépenses pour les aides à la balance des paiements fluctuent énormément d'année en année. Cela s'explique par le fait 
Tableau $\mathrm{N}^{\circ} 20$

\begin{tabular}{|l|rr|rr|}
\hline \multicolumn{6}{|c|}{ Mesures de politique économique et commerciale 1987/88 } \\
\hline & \multicolumn{2}{|c|}{1988} & \multicolumn{2}{c|}{1987} \\
\hline & mio Fr. & $\%$ & mio Fr. & $\%$ \\
\hline Aide à la balance des paiements & 40,0 & 36,8 & 25,6 & 37,8 \\
Crédits mixtes & 48,3 & 44,4 & 35,8 & 52,8 \\
Promotion commerciale & 2,6 & 2,4 & 3,0 & 4,2 \\
Promotion de l'utilisation des & 3,3 & 3,0 & 1,7 & 2,6 \\
ressources de l'économie privée & 14,6 & 13,4 & 1,7 & 2,6 \\
Matières premières & 108,8 & 100,0 & 67,8 & 100,0 \\
Total & \multicolumn{4}{|c}{}
\end{tabular}

Source: Rapport annuel de la DDA et de l'OFAEE 1988

que c'est la date du versement qui est déterminante pour les statistiques, bien qu'une longue et intensive phase de préparation ait souvent précédé ce moment. En 1988, deux accords sur l'aide à la balance des paiements ont été conclus avec Madagascar et le Ghana.

L'aide au Ghana est à la fois un cofinancement avec la filiale de la Banque mondiale, l'agence internationale pour le développement (AID), dans le cadre d'une réforme du secteur financier (15 millions de francs) et une action bilatérale de 10 millions pour le financement d'importations prioritaires. Ces moyens financiers sont mis à la disposition du système ghanéen d'enchères aux devises. Le bénéfice réalisé par cette mise aux enchères de la monnaie permet de financer des programmes sociaux pour soulager les cas difficiles engendrés par les programmes de restructuration.

Dans le cas de Madagascar, une action bilatérale de 10 millions de francs a permis le financement de produits importés pour lutter contre la malaria. Un autre versement de 10 millions s'est fait sous forme de cofinancement d'un programme de restructuration du secteur public mené par le gouvernement malgache conjointement avec la Banque mondiale.

La Suisse a participé pour 5 millions de francs en 1988 à un fonds pour le rachat des créances boliviennes, fonds administré par le Fonds monétaire international. Le rachat aux banques étrangères s'est négocié en moyenne à $11 \%$ de la valeur nominale. 


\section{Crédits mixtes}

Depuis 1977, la Suisse a garanti 24 crédits mixtes (y compris les rallonges) pour un montant total de 1'324 millions de francs. La part de la Confédération s'élève à 498 millions. Les crédits mixtes se composent d'une tranche de crédits de financements privés à l'exportation (aux conditions du marché) et d'un don de la Confédération. Jusqu'en 1987, la tranche de la Confédération sur les crédits mixtes a toujours été accordée à titre de prêt à long terme, sans intérêt. Les dispositions de l'OCDE en matière de crédits liés prévoient cet élément de don. II devrait même représenter le 25 à $35 \%$ du montant du crédit mixte accordé.

En 1988, un accord de crédits mixtes a été conclu avec la Thaïlande d'une valeur de 60 millions de francs. Deux crédits mixtes signés avec le Honduras et le Zimbabwe ont obtenu des rallonges de 0,9 million, resp. 1,2 million de francs. Des négociations ont eu lieu avec le Guatemala, la Colombie, les Philippines et le Zimbabwe. Au cours du premier semestre de 1989, un accord avec les Philippines concernant un crédit mixte de 60 millions de francs, dont 24 millions de la Confédération, a été conclu. Le Guatemala a signé un crédit mixte de 35 millions, dont 14 millions de la Confédération et le Zimbabwe a conclu son troisième accord d'une valeur de 30 millions, dont 10,5 millions de la Confédération, en mai 1989.

\section{Matières premières}

Dans le secteur des matières premières, on a été très prudent dans les dépenses, dans l'attente qu'un concept soit établi permettant un engagement intelligent des moyens de soutien. Dans le crédit-cadre actuel, 40 millions de francs sont budgetés pour les mesures de compensation lors de pertes de recettes d'exportation que les pays du Tiers-Monde subissent dans leur commerce avec la Suisse. Six programmes de compensation ont été approuvés en 1988 pour des pays africains (le Soudan, la Tanzanie, le Tchad, le Togo, l'Ouganda et la République centrafricaine) pour une somme de 15,8 millions de francs. Ces paiements compensatoires équivalent aux pertes de recettes d'exportation sur le coton, le café, les arachides et le cacao des années 1986 et 1987. On a financé des mesures de restructuration qui devraient s'attaquer aux racines du problème de la baisse des prix.

\section{Promotion commerciale}

Dans ce domaine, la Confédération soutient la Centrale suisse de promotion commerciale, qui, en plus de la promotion générale du commerce extérieur, aimerait intensifier les échanges commerciaux entre la Suisse et les pays en voie de développement. Dans ce but, elle se charge d'inciter les maisons suisses à participer à des foires commerciales dans le Tiers-Monde ou d'encoura- 
ger les pays en développement à participer aux foires commerciales suisses. Au cours de l'exercice couvert par l'Annuaire, le Parlement a voté une loi fédérale concernant une aide financière à la Centrale suisse de promotion commerciale et a approuvé un crédit de 50 millions de francs au maximum pour ces cinq prochaines années.

Pour la coopération suisse au développement, l'encouragement du commerce entre les pays en développement eux-mêmes est un des objectifs importants. La Confédération finance régulièrement des séminaires où sont expliqués les mécanismes du système des préférences tarifaires dans le but que les pays en développement y feront davantage appel.

\section{Promotion de ressources privées}

Cette mesure comprend le financement du bureau de l'ONUDI à Zurich ainsi que le soutien au projet des services de conseils et relations. Ce projet devrait faciliter le transfert de technologies et de connaissances de petites et moyennes entreprises suisses dans des entreprises en Asie pour soutenir leur développement (selon le rapport de l'économie extérieure 1988, p. 53).

\subsection{Aide humanitaire}

En 1988, 113,4 millions de francs ont été versés pour des mesures d'aide humanitaire et 58,4 pour l'aide alimentaire. L'aide humanitaire inclut l'aide en cas de catastophes (financement du corps suisse d'aide en cas de catastrophe pour 13,4 millions) et les contributions aux organisations internationales (100 millions). Le $34 \%$ des moyens a été employé pour des actions à court terme d'aide d'urgence et d'aide aux catastrophes. $31 \%$ était un soutien social à moyen terme et $35 \%$ était les contributions ordinaires aux organisations internationales et au CICR.

C'est à nouveau à l'Afrique que la plus grande partie de l'aide humanitaire a été consacrée en 1988 avec 42 millions de francs; 31 millions sont allés dans le bassin asiatique et 12 en Amérique latine.

Les contributions aux organisations internationales, qui représentent avec 100 millions plus de la moitié de l'aide humanitaire, englobent par exemple les contributions versées au Haut-Commissariat des Nations-Unies pour les réfugiés (HCR). Le nombre des réfugiés dans le monde entier est estimé à 14 millions d'êtres humains, dont la plus grande partie se trouve dans les pays du Tiers-Monde. La moitié des réfugiés se trouve en Afrique. Le HCR garantit aux réfugiés du monde entier protection et aide; il propose des solutions, comme le retour volontaire dans la patrie, l'insertion dans le premier pays d'accueil et l'établissement dans des pays tiers. Le soutien de la Suisse au HCR s'élève à 20 millions par année. 
Le Conseil fédéral a proposé dans un message de fixer la contribution annuelle au Comité international de la Croix-Rouge (CICR) à 50 millions de francs pour 1990 et 1991 et à 55 millions pour 1992 et 1993. (Ces montants correspondent grosso modo à la moitié des frais permanents d'infastructure du CICR). Le CICR est une organisation humanitaire suisse neutre et indépendante avec un champ international d'activités. Le CICR garantit aux victimes de conflits armés ou de régions à tension et à troubles assistance et protection. La Confédération participe depuis 1931 au financement du CICR.

L'aide alimentaire comprend des produits laitiers suisses (poudre de lait, fromage), des céréales et différents produits alimentaires (tels les poires séchées, les conserves de poissons, la graisse alimentaire). Au niveau de la collaboration multilatérale, la Confédération verse des contributions au programme alimentaire mondial (PAM). Le PAM est le partenaire le plus important de l'aide alimentaire suisse. Le PAM s'efforce d'acheter les produits alimentaires dans les pays en développement plutôt que dans les pays industrialisés afin de soutenir l'agriculture locale du Tiers-Monde. La Suisse soutient ces efforts, en mettant plus de liquidités à disposition pour les achats sur place.

\section{Nouveau crédit-cadre}

Depuis le 1er mars 1989, un nouveau crédit-cadre de 530 millions de francs est entré en vigueur pour trois ans. Par rapport au crédit-cadre précédent de $\mathbf{4 4 0}$ millions, l'aide humanitaire ainsi que l'aide alimentaire ont été augmentées de $20 \%$. Ce crédit a été discuté et approuvé au cours de l'exercice couvert par l'Annuaire à une large majorité. (L'édition précédente avait largement présenté le message s'y rapportant.) Le Conseil national avait approuvé le nouveau crédit sans opposition lors de la session d'automne 1988, le Conseil des Etats lors de la séance d'hiver 1988. On a pris note avec satisfaction de la modification d'option en ce qui concerne l'aide alimentaire. Selon le message, l'aide alimentaire n'est à employer que lorsqu'elle est vraiment la forme d'aide la plus adaptée. En outre, les habitudes alimentaires locales doivent impérativement être respectées lors du choix des produits de l'aide alimentaire.

\subsection{Aide multilatérale}

L'aide multilatérale est contestée dans l'opinion publique suisse. La demande a été renouvelée qu'une analyse de l'effet de l'aide multilatérale soit faite et qu'un concept soit élaboré pour cette forme d'aide. Au cours de la période couverte par l'Annuaire, la Confédération a demandé une expertise à propos des effets des projets internationaux auxquels la Suisse participe dans six pays en développement, soit au Bangladesh, au Rwanda, au Niger, à Madagascar, au Bénin 
et au Laos. Un tiers des projets a été qualifié d'insuffisant quant aux effets tangibles de l'aide sur la population. A l'avenir, cette forme de contrôle va s'institutionaliser au sein de la DDA et s'étendre à d'autres pays.

L'aide multilatérale comprend les contributions aux organisations internationales de développement. Le total de 159,3 millions de francs s'est réparti en 1988 de la façon suivante: 93,7 millions de contributions à des organisations des Nations-Unies, 40,4 millions aux banques régionales de développement et leurs fonds spéciaux et 25,2 millions à d'autres institutions multilatérales. Les données détaillées se trouvent dans le tableau 21 et le tableau 3.4 de la partie statistique.

\section{Organisations des Nations-Unies}

Le partenaire le plus important de la coopération suisse au développement multilatérale, c'est le PNUD, le Programme de développement des Nations Unies. En 1988, 51 millions lui a été attribué, ce qui représente plus de la moitié des moyens. Le PNUD finance la plus grande partie de l'aide technique du système de l'ONU. L'exécution des projets est en général menée par un des services spécialisés de l'ONU (l'Organisation mondiale de la santé (OMS), l'Organisation pour l'alimentation et l'agriculture (FAO), etc.). Le PNUD joue un rôle central dans de nombreux pays pour la planification et la coordination de l'aide extérieure. L'aide se concentre de plus en plus sur les pays les moins avancés ainsi que la promotion de l'autonomie des pays bénéficiaires, ce qui correspond aux buts de la coopération suisse au développement. Depuis la fondation du PNUD, la Suisse verse des contributions; elle est membre du conseil d'administration.

Le deuxième partenaire en importance de la collaboration multilatérale est le Fonds d'aide à l'enfance des Nations Unies (UNICEF). La Confédération a versé à cette organisation 15 millions de contributions en 1988. L'activité principale de l'UNICEF est la mise sur pied et l'amélioration des soins de base. Dans un rapport analysant les effets des réformes économiques et des programmes de restructuration sur les conditions de vie des couches les plus fragiles de la population, en particulier des mères et des enfants, I'UNICEF (3) a grandement contribué à la création de Fonds social d'urgence et de programmes de contrôle de la situation alimentaire, deux activités soutenues par la Suisse.

\section{Banques régionales de développement}

Les banques régionales de développement sont des partenaires importants de la coopération suisse au développement dans le cadre multilatéral. Leur rôle dans le processus de développement des pays du Tiers-Monde a grandi en importance au fur et à mesure que le flux de capitaux privés diminuait.

La Suisse est membre des trois banques régionales de développement et alimente aussi régulièrement leurs fonds spéciaux. En 1988, le Conseil fédéral 
Tableau $N^{\circ} 21$

\section{Contributions annuelles à des institutions internationales}

\begin{tabular}{|c|c|}
\hline Institutions & $\begin{array}{l}1988 \quad 1987 \\
\text { Versements } \\
\text { en mlo de fr. }\end{array}$ \\
\hline
\end{tabular}

Organisation des Nations Unies (ONU)

Programme des N.U. pour le développement (PNUD)

Fonds international de secours à l'enfance (UNICEF)

Fonds pour les pays les moins avancés

Fonds des N.U. pour les activités en matière de population

(FNUAP)

Fonds d'équipement des N.U. (FENU)

Programmes spéciaux de l'Organisation mondiale

de la santé (OMS)

Programme énergie PNUD/Banque mondiale

Programme Nutrition Surveillance de I'UNICEF

Organisation des N.U. pour le développement industriel (UNIDO)

Institut de formation et de recherche des N.U. (UNITAR)

Institut international de planification de l'éducation (IIPE)

Autres centres de développement des N.U.

$\begin{array}{rr}93,7 & 90,7 \\ 51,0 & 48,5 \\ 15,0 & 14,0 \\ 7,2 & 7,0 \\ 6,6 & 6,0 \\ 4,6 & 4,5 \\ 3,7 & 8,6 \\ 2,0 & 1,0 \\ 1,4 & - \\ 0,7 & - \\ 0,6 & 0,6 \\ 0,4 & 0,3 \\ 0,5 & 0,2\end{array}$

Banques régionales de développement

et leurs fonds spéciaux

Banque africaine de développement (BAD)

Fonds africain de développement (FAD)

Banque asiatique de développement (ADB)

Fonds asiatique de développement (ADF)

Banque interaméricaine de développement (BID)

Société interaméricaine d'investissement (IIC)

Fonds des opérations spéciales de la BID (FSO-BID)

Agence multilatérale de garantie des investissements (AMGI)

$\begin{array}{rr}40,4 & 27,6 \\ 3,4 & - \\ 29,0^{*} & 23,5^{*} \\ 2,1^{*} & 1,0^{*} \\ 0,4^{*} & 0,4^{*} \\ 0,5^{*} & 0,4^{*} \\ 1,1 & - \\ 1,4 & 2,3 \\ 2,5 & -\end{array}$

Autres institutions multilatérales

Fonds international de développement agricole (FIDA)

Programme Afrique du FIDA

Groupe consultatif pour la recherche agricole internationale

(CGIAR)

Union internationale pour la conservation de la nature (UICN)

$\begin{array}{rr}25,2 & 29,7 \\ 5,3 & 5,7 \\ 10,0 & 15,0 \\ & \\ 8,0 & 8,6 \\ 1,5 & - \\ 0,4 & 0,4\end{array}$

$\begin{array}{llll}\text { Conseil international pour la recherche en agro-foresterie (ICRAF) } & 0,4 & 0,4\end{array}$

Total

$159,3 \quad 148,0$

" Les contributions effectuées sous forme de "notes" (effets payables à vue) ne sont comptabilisées qu'au fur et à mesure de leur tirage.

Source: Rapport annuel de la DDA et de l'OFAEE, 1988 
a adopté un crédit global de 410 millions de francs pour les banques régionales de développement et la filiale de la Banque mondiale, l'AMGl (voir à ce sujet la discussion du message de 1986 s'y rapportant dans l'Annuaire 1986/87). Les montants accompagnés d'un astérisque ( $\left.{ }^{*}\right)$ dans le tableau 21 ont été attribués sous forme de reconnaissances de dettes ("notes"). Les tirages des "notes" sont répartis sur plusieurs années.

Banque africaine de développement (BAD): En 1987, les membres de la Banque africaine de développement s'étaient mis d'accord à propos d'une quatrième augmentation de capital et d'un cinquième réapprovisionnement du Fonds africain de développement (FAD). La Suisse a participé à l'augmentation du capital avec 250 millions de francs à titre de capital de garantie, dont 15,6 millions de francs à verser annuellement pendant cinq ans (de 1988 à 1992). Concernant le réapprovisionnement du FAD, la Confédération verse un montant non-remboursable (sous forme de bons de créances irrévocables) de $161 \mathrm{mil}$ lions de francs, à verser en trois tranches annuelles de 1988 à 1990.

Banque asiatique de développement (ADB): La Confédération a accordé à la Banque asiatique de développement un troisième crédit de 3 millions de francs en 1988 pour le financement de projets de coopération technique.

Banque interaméricaine de développement (BID): La Banque interaméricaine de développement en est toujours aux négociations concernant sa septième augmentation de capital et au réapprovisionnement de son fonds d'opérations spéciales. Une commission d'experts, mise sur pied à fin 1988, a examiné le rôle et la structure d'organisation de la banque; elle a proposé une réorganisation de la banque afin de créer une base permettant un accord pour enfin réaliser cette augmentation de capital. Selon le rapport, la BID ne s'est pas adaptée aux besoins de la région et n'a en rien contribué à résoudre la crise de la dette.

\section{Autres institutions multilatérales}

Le partenaire le plus important parmi ces institutions est le Fonds international de développement agricole (FIDA) et son Programme spécial pour l'Afrique. Le FIDA a pour but de combattre la pauvreté en milieu rural dans les pays du Tiers Monde. Son programme spécial pour l'Afrique doit permettre aux pays au sud du Sahara, menacés par la sécheresse et la désertification, d'avoir plus de moyens à disposition pour stimuler la production agricole, regénérer l'environnement naturel et promouvoir de manière générale un développement économique durable. La Confédération y a participé en 1988 avec un montant de 10 millions de francs. 


\subsection{Mesures d'ajustement structurel}

Pour les institutions financières internationales - le FMI et la Banque mondiale lla solution de la crise internationale, en particulier de la crise de l'endettement, est dans l'application de programmes sérieux de restructuration de nature politique économique. La mise en place d'un de ces programmes est très souvent la condition préalable à l'obtention d'un crédit du FMI ou de la Banque mondiale. Ces programmes ont pour but de rétablir des conditions d'équilibre normales dans les pays en voie de développement tant au niveau de l'économie extérieure qu'intérieure. Ces mesures peuvent être par exemple la dévaluation de la monnaie, la diminution des monopoles d'Etat au profit de l'économie privée, la suppression des subventions, l'introduction ou l'augmentation d'impôts.

De nombreux pays en développement se sont engagés dans cette voie d'assainissement économique. En conséquence, la coopération au développement a dû se préoccuper des mesures de restructuration et de leurs effets sur les couches les plus défavorisées de la population. II est clair que les programmes de restructuration ont des conséquences négatives à court terme sur les couches les plus pauvres de la population. Comme exemples, prenons les produits alimentaires qui renchérissent dans les villes, l'augmentation des coûts des soins de base et l'accès à la formation qui devient plus coûteux. C'est pourquoi, des programmes spéciaux ont été lancés parallèlement aux réformes afin de pallier aux nécessités sociales les plus urgentes. II s'agit entre autre de programmes d'emplois pour chômeurs et personnes sans emploi, d'aide alimentaire pour la population la plus démunie (distribution de repas dans les écoles et les fabriques), de services de soins pour certains groupes de la population.

La Suisse soutient depuis des années des programmes de restructuration et des programmes spéciaux, soit en collaboration avec la Banque mondiale/l'AID, soit au niveau bilatéral. Les pays les moins avancés disposent par exemple, grâce aux aides suisses à la balance des paiements, de devises leur permettant d'importer des pièces de rechange ou des matières premières pour des secteurs économiques en difficulté. En 1988, la Bolivie, le Ghana, Madagascar, le Mozambique et la Tanzanie ont reçu des contributions de ce genre dans le cadre de contrats bilatéraux ou de cofinancements avec l'AID.

Dans son rapport de 1988, la DDA présente des exemples de programmes de restructuration et de programmes spéciaux auxquels la Suisse participe. Les mesures de restructuration peuvent toucher tous les domaines de la coopération au développement.

\section{Madagascar}

Depuis 1981, Madagascar suit un programme de restructuration. C'est le riz qui est directement visé, l'aliment le plus important pour ce pays. Le gouvernement avait décidé de ne plus fixer le prix du riz, de libéraliser le commerce et de rendre les importations de produits alimentaires plus difficiles. Le but était de créer 
une stimulation des producteurs de riz pour qu'ils augmentent leur production. Depuis 1987, Madagascar dispose d'une production indigène de riz suffisante pour l'alimentation du pays. Mais comme auparavant, de nombreuses personnes souffrent encore de sous-nutrition et de larges couches de la population défavorisée ne disposent pas d'un pouvoir d'achat nécessaire pour se procurer des rations de riz suffisantes. En 1986, le gouvernement a créé des programmes sociaux auxquels la Suisse participe. Le riz est acheté sur place et redistribué comme aide alimentaire aux personnes les plus démunies. Parallèlement, le gouvernement a construit des entrepôts de stockage permettant de stabiliser le prix du riz tout au long de l'année. De plus, on encourage la culture de nouveaux produits et l'intensification de l'élevage de petits animaux.

\section{Mozambique}

Un rapport alarmant sur la situation économique au Mozambique avait été présenté au printemps 1987 par des représentants suisses et de l'AID. Des mesures urgentes de politique économique étaient absolument nécessaires. Le gouvernement prenait alors les décisions suivantes: dévaluation de la monnaie, augmentation des prix aux producteurs agricoles, libéralisation du commerce, suppression des subventions dans les villes sur les aliments de base - maïs, sucre, thé et savon. Quelques succès ont été enregistrés: l'offre dans les magasins et sur les marchés est devenue plus large. Les victimes de cette politique se retrouvent être les citadins vivant auparavant déjà au-dessous du minimum vital. Une étude financée par la Suisse sur la pauvreté à Maputo (capitale du Mozambique) a révélé que les aliments de base étaient devenus de trois à sept fois plus chers après la suppression des subventions dans les villes et qu'un tiers des familles (avec des enfants de 0 à 4 ans) était sous-alimenté.

"A Maputo, un tiers des habitants sont sous-alimentés. Si la paupérisation progresse encore, ces gens finiront par littéralement mourir de faim. II s'agit là de sacrifices exagérés, même si l'on espère parvenir à moyen terme à améliorer la situation. II nous faut atténuer ces souffrances. Les programmes additionnels d'assistance risquent de rendre de nouveau les villes plus attirantes et d'accélérer l'exode rural. Nous visons des objectifs qui se contredisent. Force nous est de l'avouer: nous ne disposons pas de la panacée universelle". (Rapport de la DDA, p. 13)"

Un des effets immédiats de tels programmes de restructuration est presque toujours l'accroissement de la pauvreté des couches de la population vivant avec le minimum vital. C'est pourquoi, la Banque mondiale a créé en 1988 un programme SDA (Social Dimensions of Adjustment) pour aider les pays africains les plus touchés à faire face aux coûts sociaux de tels programmes. La 
Suisse a apporté une contribution de 2 millions de francs à ce programme de la Banque mondiale.

\section{Bolivie}

La Bolivie applique depuis 1985 un programme de restructuration. Quelques succès économiques ont été enregistrés, mais les problèmes sociaux n'ont pas diminué. En automne 1986, la création d'un "Fondo social de emergencia" s'est avérée nécessaire pour atténuer les problèmes sociaux les plus criants. La Suisse a versé par deux fois 15 millions à ce fonds. En outre, des aides à la balance des paiements de la Bolivie ont été garanties qui ont permis à la Bolivie de racheter des dettes privées.

\section{L'aide privée au développement}

\subsection{Dépenses}

En Suisse, de nombreuses oeuvres d'entraide et associations de soutien, 140 environ, mènent une activité d'aide au développement. Une partie d'entre elles ont leurs propres projets et programmes, d'autres - en général les plus petites mettent la priorité sur l'information et la sensibilisation de l'opinion publique en ce qui concerne les relations de la Suisse avec le Tiers-Monde.

En 1988, les oeuvres privées d'entraide, financées par des dons privés et par la Confédération, les cantons et les communes, ont participé à l'aide au développement pour un montant total de 144,7 millions de francs (en 1987, 153,4 millions). La contribution de la Confédération a été de 109,2 millions pour des projets de coopération technique. Les mandats confiés en régie aux oeuvres d'entraide se sont montés à 74,3 millions de francs; 34,9 millions sont allés à des projets réalisés directement par la Confédération.

Le plus large soutien est allé à Intercoopération, qui n'exécute que des mandats confiés par la DDA, avec un montant de 39,3 millions de francs. Helvetas a reçu 23,6 millions et Swisscontact 11,7 millions, en partie pour des mandats et en partie pour leurs propres projets. (Les données précises sur la coopération privée au développement et sur les contributions fédérales figurent à la partie statistique de cet Annuaire.)

\subsection{Thèmes prioritaires}

Dans ce chapitre, quelques thèmes prioritaires de la coopération privée au développement sont évoqués, thèmes qui ont été abordés lors de la récolte de fonds des oeuvres privées d'entraide. 
Helvetas (Société suisse pour le développement et la coopération, Zurich) a consacré sa campagne d'information et de récolte de fonds de 1988 au thème de la femme avec le slogan "Nous les femmes, nous agissons". Les femmes ont été souvent exclues du processus de développement induit par des projets d'aide au développement qui n'étaient pas adaptés aux conditions sociales locales. L'introduction des machines dans la production agricole par exemple, un domaine qui leur était traditionnellement réservé, les a souvent exclues de la production et des revenus s'y rapportant et les a contraintes à se replier sur des activités plus domestiques. Helvetas veut à l'avenir soutenir l'initiative et la ténacité des femmes dans la perspective que seul un développement où les hommes et les femmes sont intégrés dans des projets peut avoir des chances de succès. En 1989, Helvetas a débuté une campagne d'information de trois ans sur le thème du village ("si tu veux construire ton pays, commence dans ton village"). Helvetas voit dans le développement villageois le soutien à un développement de base et une alternative aux bidonvilles des grandes cités.

La petite organisation de développement Interteam (financée par l'oeuvre d'entraide catholique Action de Carême et la Confédération) a fêté en 1989 ses 25 ans d'existence. Le service de développement catholique travaille avec le slogan "Ensemble pour un seul monde". II n'a pas de projets propres, mais il engage des gens pour des projets d'autres oeuvres d'entraide qui mettent la priorité sur la formation. Interteam s'engage aussi en Suisse pour des relations plus justes et va prendre position plus souvent dorénavant, car "l'aide au développement est sans fond, si les relations internationales ne changent pas" (Carlos Alberto Calderon à propos du travail d'Interteam).

L'EPER, qui est l'oeuvre d'entraide des Eglises protestantes", travaille dans le domaine de l'aide oecuménique, de la coopération au développement, de l'aide aux réfugiés, de l'aide d'urgence à l'étranger et de l'aide aux catastrophes en Suisse. L'augmentation du flux des réfugiés de ces dernières années a représenté une grande surcharge de travail pour cette oeuvre d'entraide. Le service d'aide aux réfugiés a passé de 6 personnes en 1978 à 65 personnes à temps plein ou partiel en 1988. L'EPER est - en plus de ses engagements dans des projets dans les pays en voie de développement - particulièrement engagée dans l'assistance aux requérants d'asile en Suisse.

La Fondation pour le village d'enfants Pestalozzi, dont l'aide est destinée aux enfants, a placé sa campagne de récolte de fonds sous le thème "nouer des réseaux de vie". La Fondation veut redonner aux enfants dont le réseau de relations sociales est détruit une nouvelle structure sociale. C'est après la Deuxième guerre mondiale que ce village a vu le jour à Trogen pour accueillir des enfants en détresse. En 1982, la Fondation a élargi ses activités à "l'aide sur place". Amener des enfants des pays en développement en Suisse au village d'enfants n'est qu'une solution de tout dernier recours. En priorité, il faut construire dans l'environnement proche des enfants une structure pédagogique et sociale adaptée.

Le service chrétien pour la paix (SCP) a été fondé il y a cinquante ans comme service d'aide aux réfugiés juifs (par Gertrude Kurz). Le SCP s'engage 
pour la paix aux côtés des êtres humains défavorisés et victimes de la répression et contre la destruction de la planète. Le SCP est une oeuvre d'entraide reconnue et subventionnée par la Confédération. Malgré tout, il est très critique par rapport à la politique officielle en matière d'asile. Les subventions fédérales ne servent qu'au soutien de réfugiés reconnus. Selon le SCP, l'assistance aux réfugiés dont les requêtes sont en suspens ou ont été refusées serait bien plus urgente ("On s'occupe des faux", Rosmarie Kurz, Tages-Anzeiger, 10.11.1988). Le SCP a évoqué la possibilité de résilier le contrat d'assistance conclu avec la Confédération afin de pouvoir s'occuper globalement des réfugiés. Le slogan de l'année jubilaire 1988 s'intitulait: "nous prenons parti". Cette prise de position en ce qui concerne les questions sociales et politiques est critique et radicalement en faveur des victimes de répression de tout ordre.

En 1988, la Déclaration de Berne (DB) a fêté ses 20 ans d'activité. La DB n'est pas une oeuvre d'entraide avec des projets propres, mais elle s'occupe avant tout d'information et de sensibilisation de l'opinion publique en matière de relations économiques entre la Suisse et les pays en développement. En 1988, un des thèmes d'information principaux a été l'endettement (elle était coorganisatrice du congrès suisse sur l'endettement de 1988). Elle a aussi montré les multiples réseaux de relations intéressées de la politique commerciale extérieure de la Suisse (édition d'une brochure intitulée "FILZ"). La DB s'est opposée à la participation de la Suisse comme membre du Club des Dix du FMI. La DB est également très critique en ce qui concerne les questions de santé et l'emploi de produits pharmaceutiques (suisses) dans le Tiers-Monde.

Depuis trente ans, Swisscontact (Fondation suisse pour la coopération technique au développement à Zurich) s'engage en matière de formation professionnelle technique dans les pays en développement. Elle a créé des ateliers d'apprentissage, des écoles, organisé des cours et encourage de manière générale l'artisanat. Swisscontact est financé par des milieux économiques suisses et reçoit un soutien de la Confédération (en 1988, la fondation a reçu 11,7 millions de francs de la Confédération et 2 millions de l'économie privée).

\section{Remarques}

1. Dans le cadre de l'OCDE, le comité d'aide au développement CAD a été créé il y a vingt-cinq ans. En font partie: l'Australie, l'Autriche, la Belgique, le Canada, le Danemark, la Finlande, la France, la Grande-Bretagne, l'Irlande, I'Italie, le Japon, la Norvège, la Nouvelle-Zélande, les Pays-Bas, la République fédérale d'Allemagne, la Suède, la Suisse et les Etats-Unis.

2. Il faut remarquer ici que la part de la Suisse calculée par l'OCDE en faveur des pays en développement est avec $0,32 \%$ du PNB plus élevée que celle indiquée par la Confédération de $0,31 \%$. La différence s'explique par une comptabilité des "notes" (reconnaissances de dettes) différente: la Suisse les enregistre au moment du paiement effectif alors que l'OCDE les enregistre au moment de leur émission.

3. UNICEF, Un ajustement à visage humain, Paris, 1988 


\section{Sources}

Rapport annuel de la DDA et de l'OFAEE 1988

Rapport sur la politique économique extérieure 1988

Rapport du Conseil fédéral sur la gestion au cours de 1988

$E+D$, Entwicklung/Développement, No. 28/1989

DFAE-communiqués de presse, 18.1., 30.3.1988, 13.3., 31.5.1989

"Welche Entwicklungszusammenarbeit für die 90er Jahre?", Andreas Schild, i3W-

Dokument 1/1989

Déclaration de Berne, rapport d'activités 1988

Helvetas Partnerschaft, sept. 1988 et 1989

Communauté de travail Swissaid/Action de Carême/Pain pour le prochain/Helvetas: prises de position 1988

Tages-Anzeiger, 10.11., 1.12.1988, 7.6., 6.9.1989

NZZ, 19.9, 1.12.1988, 23.3., 9.5., 19.5., 24.7., 15.8.1989 